\title{
Creatividad y justicia social desde un enfoque de complejidad
}

\author{
Jessica Cabrera Cuevas ${ }^{1}$ \\ https://orcid.org/0000-0002-4788-0776
}

\section{Resumen}

En este trabajo se realiza una revisión teórica de las aproximaciones de la creatividad con base en la evolución de consciencia y complejidad, para luego vincular el enfoque de complejidad con las conceptuaciones de Justicia Social Redistribución, Reconocimiento y Representación. Seguidamente, se realiza una aplicación de las investigaciones propias y ajenas en el contexto escolar, centrando la discusión en las características de escuelas que trabajan por y para la Justicia Social, como los parámetros que distinguen a las Escuelas Creativas. Finalmente, se ofrece algunas reflexiones para la acción enmarcado en escuelas de mayor vulnerabilidad dada la pertinencia de los tiempos de emergencia sanitaria global.

Palabras claves: Creatividad, Complejidad, Justicia Social, Escuelas creativas.

\section{Creativity and social justice from a complexity approach}

\section{Abstract}

In this work, a theoretical review of the creativity approaches based on the evolution of consciousness and complexity is carried out, to later link the complexity approach with the concepts of Social Justice Redistribution, Recognition and Representation. Next, an application of own and others' research is carried out in the school context, focusing the discussion on the characteristics of schools that work for and for Social Justice, as the parameters that distinguish Creative Schools. Finally, some reflections are offered for action framed in schools with greater vulnerability given the relevance of times of global health emergency.

Keywords: Creativity, Complexity, Social Justice, Creative schools.

\section{Creatividad. Aproximaciones desde la complejidad}

Para hablar de creatividad, hemos de situarnos en los nuevos enfoques de sus estudios, lo cual va más allá de definir ciertos procesos cognitivos, perfiles de personalidad, productos y ambientes que favorecen o no la misma. La creatividad hoy en día implica situarnos en el paradigma de complejidad,

\footnotetext{
${ }^{1}$ Prof. Jessica Cabrera Cuevas. Doctora en Creatividad. Docente investigadora de la Universidad Autónoma de Madrid. Contacto jessica.cabrera@uam.es.
} 
por cuanto considera los atributos no sólo a nivel de individuos, sino también de colectivos, de la sociedad y de la naturaleza.

Concordamos con las palabras de Bohm (2002), cuando señala que "la creatividad, es algo imposible de definir con palabras" (p.31), primero porque no puede encasillarse sólo en una forma particular de pensar o en perfiles que excluyen otras posibilidades de expresar los talentos o dones singulares; segundo, porque desde la Pedagogía y un enfoque humanista, nos interesa que la creatividad se desarrolle en productos que sean un aporte a la sociedad y al bien común, es decir, con sentido ético constructivo; tercero, porque la creatividad no es sinónimo de caos, ni exclusivamente de pensamiento divergente, puede darse también en el orden, la disciplina, los ritos, para llegar a resultados creativos; y cuarto, los ambientes no necesariamente son los de mayor beneficio para favorecer la creatividad, pues existen estudios que dan cuenta hace años que la creatividad también se produce y se destaca en entornos de alta resiliencia, denominada como adversidad creadora (Torre, 2009b, 2010).

En los avances del conocimiento del fenómeno de la creatividad, autores como Amabile (1996), Csickszentmilhalyi (1998) y Sternberg y Lubart (1999) incluyen en enfoque sistémico, es decir, se suma también el entorno social, denominados modelos componenciales. Por otro parte, otros investigadores en creatividad (López, 1995, Landau, 2002, Alfonso, 2006) señalan que, para explicar la creatividad, no basta con agregar factores como la motivación ni la relevancia del contexto, pues ello responde a una comprensión aún insuficiente de la creatividad. Al respecto, desde nuestro aporte, seguimos ampliando el campo de estudio de la creatividad que tiene como base la complejidad y se vincula a la evolución de la consciencia humana, por lo que concordamos en plantear la creatividad como un potencial y un valor transformador, social y ético (Torre 2006, de la Herrán, 2008) y que en su enorme diversidad y ámbitos inter y transdisciplinares, sus implicaciones epistemológicas abarcan temas tan diversos que van desde $L a$ naturaleza creativa (Moraes, 2007) a El cosmos creativo (Laszlo, 1997).

$\mathrm{Al}$ respecto, los estúdios em creatividad han ido evolucionando en complejidad, esto quiere decir que se sigue profundizando en todos sus ámbitos, como por ejemplo, el estudio de los indivíduos con altas capacidades (Blumen, 2088) nuevos estudios en torno a la intuición en la creatividad (Bejarano 2016), en los factores que intervienen en todas las personas, en el sistema en general, y más allá, como otros campos del conocimiento donde está presente la creatividad como fenómeno complejo, el cual incluye nuevos referentes transdisciplinares que incorporan las tradiciones ancestrales, 
criterios éticos, critérios de sostenibilidad, de creación de redes, y de metodologías didácticas que consideran otros campos para el saber, el aprender y el convivir (Cabrera, 2009). Por ello, para referirnos al fenómeno de la creatividad, nos basamos en un modelo que ha sido validado por expertos internacionales denominado, Modelo de tendencias en creatividad desde una conciencia compleja-evolutiva, o en su versión reducida, Modelo de tendencias en creatividad CCC, el cual es producto de una investigación que llevamos a cabo con la finalidad de aportar a la epistemología del campo de estudio de la creatividad (Cabrera, 2011).

¿Por qué incluimos el concepto de consciencia para hablar de creatividad? Cuando hablamos de que la creatividad ha ido evolucionando en complejidad de consciencia, nos referimos no sólo a un sentido teleológico y constructivo del fenómeno, sino también a la analogía producida en el desarrollo histórico de sus teorías y enfoques, por cuanto han ido integrando cada vez nuevos ámbitos de estudio en un proceso que va desde lo más particular a lo más general, es decir, una evolución que se corresponde a las dimensiones que conforma la naturaleza humana, a saber: biológica, psicológica, organísmico-social y transpersonal o espiritual. Esta evolución en el desarrollo de la consciencia humana ha sido estudiada por numerosos autores (Freud, Jung, Piaget, Freire, Rogers, Maslow, Erickson) de los cuales podemos encontrar una síntesis de sus teorías en un estudio de la Dra. González-Garza (2006) y otros estudiosos de la evolución de la consciencia.

Al respecto, el Modelo de Tendencias en creatividad CCC abarca distintos niveles de estudio que contienen sus más extensos campos aplicados. Considera en una primera subdivisión, los enfoques y teorías conocidas y consensuadas por los investigadores (en un tipo de individuos, en todas las personas y en el sistema), y luego los estudios emergentes (en complejidad). Cada una de las categorías contienen las teorías y enfoques que van desde considerar la creatividad a un tipo particular de individuos, a los estudios realizados en todas las personas incluyendo la estimulación del pensamiento creativo, la medición mediante test, las valoraciones a los productos, el desarrollo de la personalidad creadora y el campo de la psicología humanista. No obstante, los modelos componenciales incorporan el valor del ámbito de aplicación de la creatividad, incorporando lo social. Finalmente, se produce una ruptura disciplinar que profundiza aún más en el fenómeno de la creatividad con disciplinas menos convencionales, como la física, la química, la biología y la filosofía, entre otras, e integrando otros saberes vinculados a valores éticos y sostenibles como: consciencia ecológica, educación inclusiva y 
universal, la competencia de género, la evolución humana, y una creatividad con sentido dinámico, interactivo, sistémico y complejo (Cabrera, 2018).

La complejidad en la creatividad va más allá de ser un adjetivo de las personas, procesos o productos y ambientes, sino que integra, como señalan algunos científicos (Maturana, Varela, D’Àmbrosio, Prigogine, Bohm, Laszlo, Capra, Sheldrake, Mandelbrot entre otros) otras aportaciones a esta comprensión compleja y trascendente de la creatividad (en Cabrera, 2011). Esta gama multidimensional hace que haya mayor consenso y respeto a la diversidad en la enseñanza de la ética para la humanidad (Morin, 2004). En tal sentido, interesa enfocar el para qué de la creatividad, que no será otro, como plantean de la Herrán (2003) y Torre (2006), que una creatividad para la mejora personal y social. Algunas de los enfoques en esta línea de complejidad lo hallamos en las megatendencias, el enfoque transpersonal trabajados por autores como Wilber, Ferrer, Rubia, Almendro, GonzálezGarza, el pensamiento complejo de Morin (2004), enfoque ecosistémico (Moraes, 2007), Teoría interactiva y psicosocial (Torre, 2006), Teoría complejo evolucionista o de complejidad evolutiva (de la Herrán (2003, 2006), entre otros.

El Modelo de Creatividad CCC ha tenido algunas aplicaciones en contextos educativos como en la reorganización de competencias genéricas (Morales \& Cabrera, 2012); en proyectos de Aprendizaje-Servicio (Cabrera, 2015 , 2016a y 2016b) y aplicado en un proyecto educativo validado por el Ministerio de Educación de un colegio de Lisboa, Portugal en 2015, donde se abordó en ese momento como Pedagogía 3C, Consciencia, Complejidad y Creatividad (Cabrera \& Saldanha, 2017). Finalmente podemos afirmar que, en este avance de conocimiento, la creatividad desde la complejidad constituye un fenómeno que tiene sus implicaciones en la trascendencia, el bien común, la evolución basada en la consciencia, la justicia social y el cuidado del planeta.

\section{Complejidad y Justicia Social}

Desde una perspectiva de complejidad, lo social no puede ser concebido desde una simplificación reduccionista, sino más bien se concibe con derivaciones epistemológicas que van más allá de marcos puramente disciplinares particulares (como la economía, la sociología, la antropología, la historia y las ciencias políticas), contemplando un espacio multidimensional de la concepción de la realidad y del acto de comprender los procesos que subyacen en ella. En tal sentido, las sociedades contemporáneas se vuelven 
complejas por los diversos actores sociales que se relacionan a escala macro (planetaria), mezo (regional-nacional) y micro (territorial-local-comunitario familiar), además de tantos otros procesos sociales como el avance tecnológico, las amenazas medioambientales, el consumismo, la contaminación (Espina, 2004) en resumen, la necesidad de una mayor consciencia de valores éticos y sustentables.

Autores como Hessel y Morín (2013) afirman en sintonía a la mirada compleja del crecimiento, en tanto se determine que hay un crecimiento necesario, pero otro que debe decrecer, como, por ejemplo, reducir la agricultura industrializada, las energías fósiles y nucleares, los daños del consumismo y de la economía de bienes superfluos. Por ello, el tema ético es un eje que los seres humanos debemos atender para una evolución consciente y lo consideramos no sólo como una mera reflexión, sino como plantea Delgado (2011), como una responsabilidad social y colectiva orientada al futuro, dando valor a una ciencia más humanista, con valores que integran los fenómenos espirituales en la cognición, la tolerancia cultural, la justicia social y la diversidad en contextos humanos y naturales.

Concordamos además con la investigadora Moraes (2020) en que somos una comunidad global interdependiente, y que muchas de los entornos vulnerables son productos de la falta de condiciones básicas para una vida digna, la cual nos exige comprender la crisis sistémica que estamos viviendo, las causas de las injusticias sociales y falta de sustentabilidad ecológica que vivimos a nivel global y planetario. Desde el ámbito educativo, será relevante orientar todos los esfuerzos para promover una mayor justicia social.

Para dar una aproximación a la noción de Justicia Social, nos encontramos tres concepciones recurrentes: Justicia Social como Distribución, orientada en la distribución de bienes, recursos materiales y culturales, y en las capacidades la segunda, la Justicia Social como Reconocimiento, orientada al respeto cultural de todas las personas y las relaciones sociales justas y la tercera, la Justicia Social como Participación, orientada a que las personas participen en las decisiones de la sociedad de la cual forman parte de una manera activa y equitativa (Murillo y Hernández, 2011).

En la Justicia Distributiva (Rawls 1971) se encuentran los principios de Justicia igualitaria (a cada persona una parte igual); Justicia según la necesidad (a cada persona de acuerdo con sus necesidades individuales); Justicia según el mérito (a cada persona según su propia contribución a la generación de beneficios sociales), donde agrega luego la Justicia según el principio de la diferencia (Rawls, 2001) la cual se entiende que es un principio de compensación en beneficio de las personas menos aventajadas. 
En lo que se refiere al enfoque de las capacidades, introducido por Amartya Sen, plantea que no basta con una justicia basada en la distribución de recursos económicos, sino, que también es indispensable considerar la distribución de capacidades en entornos que se promuevan como justos, es decir, la idea de justicia estará ligada a las verdaderas libertades que tengan las personas para elegir las formas de vivir y convivir. Cabe destacar, que el pensamiento de este autor considera además que cuando hablamos de justica social y promover una mejora ante las desigualdades, importa atender todo tipo de dominaciones culturales, además de las económicas y de capacidades, las que tienen que ver con las diferencias de género, sexualidad, étnicas o cualquier grupo minoritario que se excluya en derechos de oportunidades y reconocimiento social y cultural (Sen, 2000, 2010).

La Justicia como Reconocimiento, es la que tiene que ver con esta ausencia de dominación cultural y como plantean Murillo y Hernández (2011) "la valoración de las minorías étnicas, raciales y sexuales intentan desarrollar un nuevo paradigma de la justicia que sitúa al reconocimiento en su centro" (p.17). Por su parte, Fraser (2008) concuerda que no sólo se trata de injusticia cuando hay una inconveniente distribución de recursos o capacidades, sino además en la falta de reconocimiento de la identidad de un colectivo determinado, por cuanto al no ser reconocidos, son oprimidos por los grupos más privilegiados.

Por último, la Justicia como Participación o Justicia de Representación (Murillo y Hernández, 2014) implica la equidad en la participación de todas las personas, sin exclusiones de ningún tipo de diferencias (etnia, edad, género, habilidad física o mental, educación, orientación sexual, situación socioeconómica, entre otras) (Harnett, 2001). Al respecto, destacamos la visión que plantea Honneth (2007) por cuanto una gran injusticia es la falta de participación democrática, el autor lo considera una minusvaloración a las personas pues les excluye de derechos que otorga la sociedad en igualdad para toda la ciudadanía.

Es un hecho de que existe un deseo profundo de una mayor Justicia Social aún necesaria y urgente. Uno de los redactores de la Declaración de los Derechos Humanos de 1948, quien estuvo internado en campos de concentración durante la Segunda Guerra Mundial, es el exdiplomático Stéphane Hessel. Él vino a dar un impulso en materia social, al tener gran resonancia social con su libro ¡Indignaos! (Hessel, 2010). En él hace un llamamiento para que los jóvenes principalmente se rebelen de forma pacífica contra los poderes imperantes. Como podemos observar, la justicia social, trasciende el concepto tradicional de justicia legal, pues incluye los elementos 
esenciales respecto a la igualdad de oportunidades y de derechos humanos fundamentales y nos invita a clarificar los criterios que son socialmente justos y de bien colectivo (Montané, 2013).

$\mathrm{Al}$ respecto, hemos de considerar la gama de experiencias humanas y las emergencias que nacen de una sociedad en constante movimiento y desarrollo, por ello no podemos estar más de acuerdo con el trabajo de Boaventura de Sousa Santos que propone una ecología de los saberes (Santos, 2009) por sobre sociedades y culturas que pretenden imponer sus parámetros y valores como únicos y válidos. Esto también es justicia, una justicia al conocimiento.

Concordamos con Montané (2013) cuando señala que debemos tomar en cuenta la Educación como un bien público donde las políticas sociales y políticas educativas tienen su base en la ética y la justicia social.

\section{Comunidades educativas justas y creativas}

La educación es uno de los ejes fundamentales para una transformación social, más justa, equitativa e inclusiva. La realidad actual respecto a la pobreza, desigualdad y segregación escolar aún nos dan cifras alarmantes a nivel mundial. Haciendo una analogía, la escuela puede ser como una partícula que reproduce los componentes de la sociedad. En ella encontramos elementos intrínsecamente vinculados a los problemas sociales, como situaciones y problemáticas comunes en materia de multiculturalidad, temas de género, desigualdades económicas, de rendimiento, de relaciones sociales, de exclusión, entre muchas otras. En tal sentido, se hace pertinente orientar como una finalidad esencial el favorecer una mejora en las desigualdades que se presentan en una comunidad educativa y promover escuelas con un funcionamiento y organización más justa, democrática y creativa.

En una educación para la Justicia Social, donde la escuela con toda su comunidad educativa participe, tanto docente, familia y alumnado, los autores Murillo y Hernández (2014) consideran los siguientes cuatro indicios: primero, algunas escuelas contribuyen a la reproducción de desigualdades y otras a la transformación social, y esto tiene que ver con la responsabilidad personal de cada integrante de la comunidad, como actor participante de injusticias o como observador indiferente, por lo tanto, implica un compromiso para visibilizar situaciones injustas que tengan impacto en la comunidad y sociedad; segundo, para promover una sociedad más justa, la escuela debe plantearlo explícitamente, esto implica una declaración de 
principios en los proyectos educativos que harán que no sólo sean discursos de buena voluntad sino que para no replicar las desigualdades sociales, la escuela lo debe plantear en sus objetivos y plan de acción; tercero, una escuela que trabaja por la Justicia Social y la equidad escolar lo revelará en su visión y en sus acciones cotidianas, esto quiere decir que se promuevan las capacidades y competencia para modificar situaciones injustas y que el ambiente de la comunidad educativa comparta una cultura donde se trabaje en coherencia para, en y desde la Justicia Social; cuarto, para promover un compromiso por la Justicia Social, la escuela contará con un buen estilo de liderazgo. Al respecto el liderazgo escolar para la Justicia Social es un enfoque teórico práctico orientado a una educación y sociedad más justa, y democrática, no puede haber participación desde una organización autoritaria, por cuanto, se vuelve una condición imprescindible que la escuela funcione desde la democracia.

Para una Educación en Justicia Social los autores destacan algunos elementos: autoconocimiento y autoestima del estudiantado respecto a sus identidades y cultura; respeto a los demás creando un clima de respeto y empatía a la diversidad; abordar situaciones de injusticia social en diversos colectivos como el sexismo, clasismo, homofobia e intolerancia y opresión de cualquier tipo; promover el movimiento y cambio social a través de ejemplos de personas destacadas y el trabajo conjunto; despertar la consciencia a través de la sensibilización de temas relevantes para la comunidad; correspondencia con la acción social en situaciones concretas de la comunidad, por ejemplo, en situaciones de exclusión por capacidades, nivel socioeconómico, cultural u origen entre otras.

Según los componentes de Justicia Social antes mencionados de Redistribución, Reconocimiento y Representación (o participación), destacamos algunos ejemplos (Tabla 1) de lo que serían estos componentes en las escuelas que integran la Justicia Social.

Tabla 1 : Componentes de la Justicia Social aplicado a las Escuelas

Componentes de Justicia Social

Características de las Escuelas Justas

Redistribución

Una escuela se considera justa cuando toda su comunidads compromete aprendiendo con procesos de enseńanza aprendizaje que sean justos

\footnotetext{
. Objetivos explícitos conocidos por la comunidad,

. Conseguir el máximo desarrollo de los estudiantes

- Valores y actitudes que fomentan la inclusión, la autoestima y bienestar de los estudiantes

Favorecen la equidad para los más desfavorecidos y vulnerables

. Promueven una cultura donde se fijan metas altas ofreciendo oportunidades para alcanzarlas

. Mantienen una actitud solidaria y de apoyo mutuo

. Promueven el sentido de pertenencia
} 


\section{Reconocimiento}

La escuela promueve una cambio cultural y social a través de una educación en valores que reconozca las identidades y productos culturales de su comunidad respetando la diversidad según género, cultura, etnia, orientación sexual ...
. Compromiso colectivo entre la labor docente curricular . Aprendizaje colaborativo

. Abordaje de la innovación y nuevos retos

. Promueven desarrollo integral del estudiante y el desarrollo de la creatividad

. Propicia el pensamiento crítico y valores democráticos

. Atención responsable de la diversidad desde la enseñanza, metodologías y evaluaciones

. Promover un currículo que atienda a temas de género, cultura, etnia y equidad

. El profesorado valora la diversidad y promueve su reconocimiento social

. Se valoran las diferentes tradiciones culturales y se promueve un Curriculum multicultural

. Se favorece el pensamiento crítico y razonamiento ético junto a la denuncia de situaciones injustas

. Se promueve la responsabilidad individual y la solidaridad entre valores sociales diversos y la interdependencia

. Se valora las diferencias culturales y lingüísticas de las familias como un valor que amplía los aprendizajes para la clase

. Se valora la colaboración entre la escuela y las familias para compartir la cultura educativa desde el respeto y la colaboración conjunta

\section{Representación}

La escuela trabaja para promover el compromiso y participación de toda la comunidad escolar a nivel de organización escolar y proyecto educativo curricular
. Se fomenta una cultura de respeto

. Los estudiantes participan activamente de su formación, especialmente los colectivos tradicionalmente marginados

. Se promueve una cultura democrática en la toma de decisiones didácticas

. Se contempla la participación de toda la comunidad, docentes, familias, estudiantes, personal no docente . Reuniones periódicas del consejo escolar y el claustro . Se promueve un liderazgo distribuido en la comunidad . Se fomenta la apertura al entorno a través de los propios barrios y asociaciones locales

Fuente: Murillo y Hernández, 2014, adaptado.

Para las escuelas que trabajen por y para la Justicia Social y superar las desigualdades, el tipo de liderazgo es fundamental para trabajar realmente el cambio cultural de la escuela, promover la inclusión, la colaboración con las familias y proyectar la comunidad donde sus miembros se sientan orgullosos de pertenecer. Por tanto, el equipo directivo será clave en las decisiones que se tomen a nivel del centro, y decimos equipo, pues no tiene que ver con un 
cargo específico, sino más bien con un colectivo que comparten el compromiso y colabora en las iniciativas que responden a favorecer su comunidad más justa.

Los líderes que promueven y consiguen que una escuela esté alineada a los valores de Justicia Social se comprometen con algunas acciones como; entusiasmar y trabajar el cambio cultural de la escuela; potenciar el desarrollo personal y colectivo de su comunidad, favorecer procesos didácticos eficaces desde la Justicia Social; promover la colaboración de las escuelas, la familia y el entorno, así como el valor de la multiculturalidad (Murillo y Hernández, 2014). A nivel de equipos directivos que trabajan en y para la Justicia Social, diversos autores (Salisbury y McGregor, 2005 apud Murillo y Hernández, 2014) concuerdan en que estos equipos comparten objetivos como: son más accesibles, se interesan e involucran en resolver problemas; invierten en la relaciones y convivencia de cada miembro de la comunidad en primera línea; generan un clima de confianza; asumen riesgos; se comprometen con soluciones innovadoras; ponen intención y reflexión para conseguir los objetivos, tomando en consideración para conseguir las mejores decisiones; promueven la trasparencia y la humildad para la toma de decisiones y analizar las consecuencias.

En consecuencia, para que haya una escuela que apueste por y para la Justicia Social, es necesario un liderazgo ético, comprometido a valores democráticos y distribuido, comprometido, colaborador y motivado en contribuir con una sociedad más justa desde esta micro sociedad que es la escuela.

Por su parte, las Escuelas Creativas, según los fundamentos de la Red Internacional de Escuelas Creativas RIEC, comparten la mayoría, sino todos, los postulados de las Escuelas que trabajan en y para la Justicia Social, dando valor a la creatividad desde un enfoque Transdisciplinar, la Ecología de los Saberes y la Ecoformación. Esto implica una mirada ecosistémica sobre la educación, que integra además de los valores sociales, otros que tienen que ver con lo transpersonal y trascendente.

En esta corriente transdisciplinar que incluye el valor de la consciencia y la ética, aparecen científicos de diferentes disciplinas (Maturana, Varela, Binning, Sheldrake, Bohm, Prigogine, Laszlo, Morin, Moraes, DÀmbrosio, Santos, entre tantos otros) que, sin embargo, rompen su cerco epistemológico de conocimiento abriéndose a una actitud transdisciplinar y compleja. Las tres grandes vertientes de la transdisciplinariedad serán primero la vertiente epistemológica y metodológica y visión cuántica de la realidad -o realidades-; la vertiente enactiva o de acción informada que se proyecta en la formación y 
ecoformación; la vertiente actitudinal y de compromiso con los otros y lo otro. La Ecoformación en resonancia a esta visión, propone un crecimiento interior desde lo multidimensional, partiendo del respeto a la naturaleza (ecología) considerando los otros (alteridad) y trascendiendo la realidad sensible (transpersonalidad), creando espacios colaborativos y con ambientes amables de trabajo, estrategias dinámicas, flexibles, innovadoras y que generen motivación Por tanto, estamos ante un paradigma profundamente humanista, social y ecológico-sostenible, basado en valores humanos, en el desarrollo de la consciencia, en la creación de redes y campos de emprendizaje (Torre, Pujol y Sanz, 2007).

La sociedad del conocimiento se ha mostrado parcialmente injusta, excluyente y aval de saberes útiles a la productividad y competitividad. Acordamos con Raúl Motta, cuando plantea que "Educar con otra consciencia es cuestionar la organización de los saberes y conocimientos establecidos, sobre la base de la constatación de que no coinciden, ni son tal como están, accesibles para la resolución del orden y la prioridad de los problemas a los que las sociedades del presente se enfrentan cotidianamente" (Motta, 2009, p. 63-64). Por ello el desafío transdisciplinario se debate entre la necesidad de una visión unificada de la realidad y la creatividad científica, artística, cultura y tecnológica, la emergencia de un sujeto con consciencia planetaria y la democratización del conocimiento y la relación de sus paradigmas.

Para educar con una consciencia de creatividad, nos vemos llamados a acercar y abrir la escuela a la vida, la sociedad y la naturaleza de la cual formamos parte, para priorizar valores, actitudes, hábitos y competencia por encima de los conocimientos académicos (Torre, 2009a). Todo ello cobra especial relevancia en este tiempo de pandemia, donde las escuelas han pasado abruptamente a reconfigurar sus programaciones, algunas con muchas limitaciones y aplicando la didáctica al entorno inmediato de las familias y sus educandos.

Para un Curriculum del siglo XXI se considera el triángulo de la vida D’Ambrosio tomando en cuenta la autoformación (centrada en sí mismo) la heteroformación (influencia de la sociedad) y la ecofroamción (influencia de la naturaleza para una educación sostenible) y donde aún sigue vigente el informe Delors et al (1997) a través de la ciencia (aprender a conocer), la tecnología (aprender a hacer), la convivencia y diversidad (aprender a convivir) y la educación integral (aprender a Ser), es decir, un estudio basado en el medio físico, social, (que incluye lo político y económico) y lo espiritual (Mallart y Mallart, 2013). Se hace necesario, por tanto, pensar una propuesta educacional que favorezcan los diversos aprendizajes con estrategias didácticas 
creativas, innovadoras y enriquecedoras, que promueva la interrelación de las percepciones, las emociones, intuiciones y la cognición para comprender en mayor profundidad la multidimensionalidad de la condición humana integrando cuerpo, mente y espíritu. Una escuela que promueva el autoconocimiento, el reconocimiento del otro, el desarrollo de la sensibilidad, de la escucha sensible y de la autoestima, nutrida por la ética y estética del pensamiento, la mirada holista que implica una mayor responsabilidad y solidaridad (Moraes, 2015).

La propuesta de Escuelas Creativas fue promovida por Saturnino de la Torre, hoy catedrático emérito de la Universidad de Barcelona, en el año 2009, y creada formalmente en el año 2012 en la Universidad de Barcelona con investigadores internacionales en la que participamos, y posteriormente, ha sido inscrita como Asociación de Escuelas Creativas ADEC, en el Ministerio del Interior en 2018. Está constituida por más de una veintena de Núcleos de trabajo en España y Brasil, como el Núcleo RIEC-UAM-Madrid, a través del cual se ha firmado un convenio de colaboración con la Cátedra Unesco en Educación para la Justicia Social de la Universidad Autónoma de Madrid, España en 2019.

A través de la red de investigadores e investigadoras de la RIEC, se valora la conexión y retroalimentación de las instituciones educativas que se interesan por el valor de la creatividad y prácticas innovadoras. Por ello, la RIEC pretende crear una red de comunidades educativas donde intervenga la investigación académica, las administraciones y las escuelas comprometidas con el desarrollo de la consciencia los valores humanos, sociales, éticos y sostenibles. Para ello se elaboró un instrumento que fue presentado con ocasión del IV Forum Internacional sobre Innovación y Creatividad. Adversidad y Escuelas creativas realizado en Barcelona en junio de 2012 donde participamos en su validación interna (Torre, 2012). Este instrumento para Valorar el Desarrollo Creativo de las Instituciones Educativas, VADECRIE, se pone al servicio de las escuelas que quieran valorar y reconocer el potencial creativo de su comunidad, como una referencia para el autoanálisis institucional. Dicho instrumento cuenta con diez (10) parámetros con, a su vez, diez (10) categorías cada una con sus respectivos descriptores para medir: Liderazgo estimulador y creativo; Profesorado creativo; Cultura innovadora; Creatividad como valor; Espíritu emprendedor; Visión transdisciplinar y trasformadora; Curriculum polivalente; Metodología Innovadora; Evaluación Formadora y transformadora; Valores humanos, sociales y medioambientales. 
Las Escuelas Creativas se caracterizan como aquellas instituciones que trascienden (van más allá del lugar en que parten) recrean (da más allá de lo que de ellas se espera), valoran reconocen lo mejor de sus estudiantes y profesores) y transforman (crecen por dentro y por fuera buscando la mejora), (Torre, 2013). Además de potenciar la creatividad, el diálogo y la colaboración, la propuesta de Proyectos Creativos Ecoformadores, PCE, se enfocan en potenciar la enseńanza a partir de la vida, con soluciones solidarias y sostenibles. Representan una referencia para un aprendizaje basado en la autonomía, la transformación y la colaboración y desarrollo integral; partir desde los intereses de los estudiantes, estimular un práctica flexible y abierta a emergencias; fomentar la resiliencia y adversidad creadora (Torre \& Zwierewicz, 2009).

Nos encontramos en un momento de grandes desafío y adversidades en las escuelas a escala global a propósito de la pandemia, por lo que la creatividad se reconoce como urgente para la reingeniería que tantas escuelas han debido abordar. Cobra sentido y pertinencia destacar el papel de la creatividad, y de la adversidad creadora (Torre, 2011), la cual permite a los estudiantes, docentes y toda la comunidad, tomar consciencia del potencial creativo que tienen las situaciones adversas, y poder transformar la adversidad con acciones creativas y éticas (Suanno y Cardoso, 2013). Es preciso que los educadores puedan reflexionar e investigar acerca de sus propias prácticas, sus valores y concepciones ideológicas, pedagógicas y valóricas para poder fortalecer la resiliencia necesaria para la superación de las adversidades. Cobra sentido articular la autoformación, heteroformación y ecoformación para estar mejor preparados para los momentos actuales y futuros.

Desde el Modelo de Creatividad CCC, que ordena el mapa de los estudios y aplicaciones de la creatividad a nivel de individuos, personas, sistema y complejidad, hemos desarrollado la propuesta que integra los tres ejes y que denominamos, Modelo Potencial 33: Consciencia, Complejidad y Creatividad, y que se propuso en una ordenación de los parámetros del instrumento VADECRIE a nivel teórico, personal y estratégico, a saber: Consciencia, se refiere a los planteamientos epistemológicos (principios, valores, teorías, visión, misión del proyecto educativo); Complejidad (implica las interrelaciones del potencial humano o comunidad educativa; Creatividad (implica el potencial transformador del currículo y el plan estratégico de acción). Por tanto, los parámetros para orientar el diagnóstico de las escuelas (Torre, 2012 p.155) quedan como se muestra en la Figura 1. 
Figura 1. Agrupación de parámetros de VADECRIE según el Modelo Potencial 33.

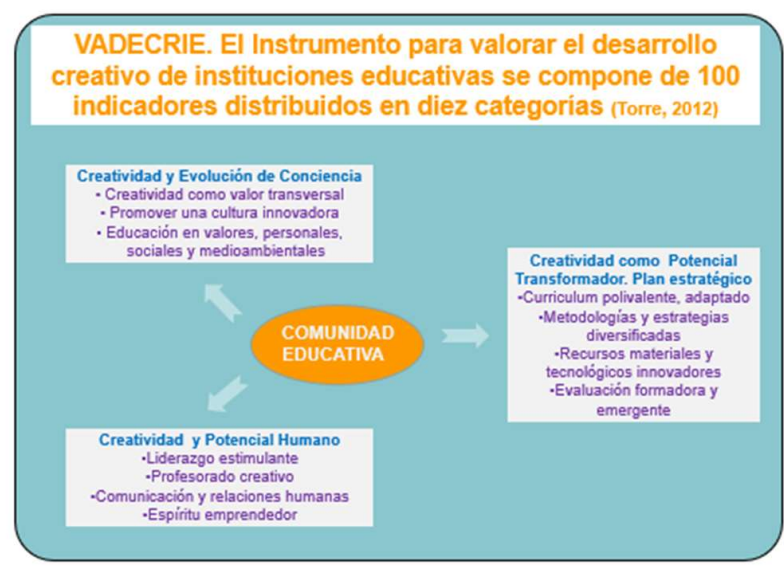

Consciencia, complejidad y creatividad

Por otra parte, en la implementación de un Proyecto de Innovación en la Universidad Autónoma de Madrid (INNOVA FPE_018.18_INN) para la "Implementación de la Red Internacional de Escuelas Creativas, RIEC Núcleo Madrid", se aplicaron tres cuestionarios de VADECRIE (Cuestionario para evaluar el profesorado, el equipo directivo y la cultura innovadora) y en los cuales ha tenido pertinencia la utilización del Modelo Potencial 33 consciencia, complejidad y creatividad en el análisis de las categorías de cada ítems, según tuvieran que ver con la epistemología del proyecto educativo, la comunidad educativa o el plan metodológico estratégico. De esta manera, puede ser de gran utilidad para el inicio del apoyo e intervención para la escuela y su sello como escuela creativa.

Con esta propuesta, podemos promover un impacto favorable en las comunidades educativas, toda vez que se replantee una renovación que afecte a todo el sistema: su misión y visión, (como escuela creativa, justa y democrática), su comunidad (directivos, estudiantes, familias y resto de integrantes), sus estrategias metodológicas (aprendizaje basado en proyectos, ApS, otras), sus recursos (espaciales, tecnológicos, materiales), sus actividades, sus conexiones en red, etc. Para ello es necesaria la participación de toda la comunidad, tanto en formación, como en el diseño e implementación de sus proyectos de innovación educativa. 
Desde una cultura escolar que promueva la Creatividad y la Justicia Social, se proponen algunas orientaciones prácticas a manera de ejemplos:

a. provectos didácticos en los contextos locales vinculados a las tradiciones culturales, artísticas, la contingencia social, económica y política, ámbitos espirituales, entre otros, que permitan generar aprendizajes significativos y conscientes;

b. promover la reflexión en torno al uso de las tecnologías de la información y comunicación y recursos tecnológicos de manera adecuada tanto para lo educativo como para el ocio;

c. incorporar temáticas de pedagogía perenne, como la educación de la consciencia, pedagogía de la muerte, pedagogía prenatal, pedagogía de la ternura, entre otras propuestas sensibles y trascendentes;

d. trabajar metodologías artísticas y deportivas que favorezcan la ampliación de consciencia como la meditación, las artes marciales, el ajedrez y magia mental;

e. generar proyectos que integren la economía colaborativa y actividades solidarias al interior de la comunidad como hacia el entorno social, como son los proyectos de ApS, proyectos de emprendizaje vinculados a generar una mayor calidad de vida en la comunidad;

f. promover proyectos con una creatividad consciente a las necesidades del ecosistema, mejorar hábitos de consumo, de alimentación, de uso de recursos materiales;

g. generar una comunidad donde todos sus integrantes se sientan motivados, alegres, comprometidos y orgullosos de pertenecer;

h. promover el potencial humano y generar redes de colaboración con otras comunidades locales e internacionales;

i. promover la investigación y la innovación integrando universidad, instituciones públicas y la comunidad educativa.

\section{Aproximaciones finales}

Como hemos visto, la creatividad no sólo se puede abordar desde el nivel de la persona, sino que toman relevancia los contextos donde se produce, no sólo a nivel de ambientes que favorecen sino también en entornos de resiliencia y adversidad creadora (Torre, 2011). Adaptarse y transformar las adversidades para responder creativamente a la crisis, considerar la ecoformación, y atender a la justicia social son ejes fundamentales en los 
desafíos presentes y el porvenir. Hemos de replantearnos creativamente el universo escolar, el papel de la creatividad en sus fundamentos, metodologías, espacios, relaciones y en su conexión con el entorno y con redes de colaboración. Apostamos por una creatividad que promueva valores de paz y justicia social, que genere pasión por aprender, competencias personales que los preparen para el presente y futuro, que promueva el entusiasmo por recrearse a sí mismo y aportar bienestar al entorno. Podemos finalmente, aventurarnos a que la creatividad se vincule a una evolución de consciencia (Cabrera, 2018), esto implica, favorecer una formación que se ocupe de promover valores en torno a la consciencia de sí mismo, la consciencia social y la consciencia planetaria.

\section{Referências}

ALFONSO, V. Creatividad en la psicología. Creatividad como fenómeno complejo y multidimensional. En S. de la Torre y V. Violant (Coord.), Comprender y Evaluar la Creatividad, Vol. I,. Málaga: Aljibe, 2006. 323-333

AMABILE, Teresa. The Social Psychology of Creativity. Editor, Westview Press, 1996.

BOHM, D. Sobre la creatividad. Barcelona: Kairós, 2002.

BEJARANO, V. La intuición creadora: implicaciones y aplicaciones en la educación creativa. (Tesis doctoral), Universidad Complutense de Madrid, 2016.

BLUMEN S. Motivación, sobredotación y talento: un desafío para el éxito. Revista de Psicología, 26(1), 2018. 147-184.

CABRERA, J. Creatividad hoy. Una evolución hacia mayores niveles de conciencia y complejidad. Revista Educación y Futuro (21) 15-42, Madrid, 2009.

CABRERA, J. Creatividad, conciencia y complejidad. Una contribución a la epistemología de la creatividad para la formación. Tesis doctoral. Universidad Autónoma de Madrid, 2011.

CABRERA, J. Creatividad, complejidad y formación: un enfoque transdisciplinar. Revista Complutense de Educación. Vol. 26 Núm. 3, 2015. 505-526 
CABRERA, J. Tendência transdisciplinares: um enfoque favorável para a formaçao emcriatividade e a criatividade para a formaçcao. En Innovaçao e Educaçao. Formaçao docente e experiências criativas. Santos, Lorenzo \& Regina (Orgs.) Editora Unijuí. Ijuí, Brasil, 2016a.

CABRERA, J. La práctica del ApS en alumnos de magisterio de primaria y el reforzamiento de competencias genéricas según una propuesta de complejidad y transdisciplinariedad. En El Aprendizaje-Servicio en las Universidades. De la Iniciativa individual al apoyo institucional. Aramburuzabala, P. Opazo, H. y García-Gutierrez, R. (Coords.). UNED, 2016b.

CABRERA, J. \& SALDANHA, I. Pedagogía 3C. Un modelo de innovación educativa aplicada. En Actas de Congreso VIII Fórum Internacional D’Innovació i Creativitat. Compartint Experoències Innovadores i Creatives en Educació. Universidad de Barcelona, 2017.

CABRERA, J. Epistemología de la creatividad desde un enfoque de complejidad. Revista Educación y Humanismo, 20 (35), 2018. 113-126.

CSIKSZENTMIHALYI, M. Creatividad: El fluir y la psicología del descubrimiento y la invención. Barcelona: Paidós, 1998.

DELGADO, C. Hacia un nuevo saber. La bioética en la revolución contemporánea del saber. Ed. Acuario, Centro Félix Varela, La Habana, 2011.

DELORS, J., AMAGI, I., CARNEIRO, R., CHUNG, F., GEREMEK, B., GORHAM, W., ... \& Stavenhagen, R. La educación encierra un tesoro: informe para la UNESCO de la Comisión Internacional sobre la Educación para el Siglo Veintiuno. UNESCO, 1997.

ESPINA, M. Complejidad y pensamiento Social. Gestión de las Transformaciones Sociales MOST. Documento de debate, No 70. En Transdisciplinariedad y Complejidad en el Análisis Social. Luis Carrizo, Marya Espina Prieto \& Julie Klein, UNESCO, MOST, 2004.

FRASER, N. Scales of Justice: Reimagining Political Space in a Globalizing World. Nueva York: Columbia University Press. Traducción al español. Escalas de justicia. Barcelona: Herder, 2008.

GONZÁLEZ-GARZA, A. Ma . El desarrollo de la consciencia desde la óptica transpersonal. En M. Almendro (Ed.), La consciencia transpersonal (2a ed.) 315-351. Barcelona: Kairós, 2006. 
HARTNETT, D. The history of justice. Paper presented at the Social Justice Forum, Loyola University, Chicago, 2001.

HESSEL, St., Morín, E. El camino de la esperanza. Buenos Aires: Editorial Paidós, 2013.

HERRAN, A. de la. El Nuevo Paradigma Complejo-Evolucionista. Revista Complutense de Educación, 14 (2), 499-562. Madrid, 2003.

HERRÁN, A. de la. "Hacia una creatividad complejo-evolucionista. Redefinición del concepto de creatividad desde una educación de la conciencia”. En S. de la Torre y V. Violant (Coords.), Comprender y Evaluar la Creatividad, Vol. I. Málaga: Aljibe, 2006. 61-68.

HERRÁN, A. de la Didáctica de la creatividad. En A. de la Herrán y J. Paredes (Coord.) Didáctica General. La práctica de la enseñanza en Educación Infantil, Primaria y Secundaria. Madrid. Mc Graw Hill, 2008. 151-176.

HESSEL, S. Indignaos. Barcelona: Destino, 2010.

HONNETH, A. Reificación. Un estudio en la teoría del reconocimiento. Madrid: Katz, 2007.

MALLART, J. \& MALLART, A. Ecoformación y Transdisciplinariedad. Fundamentos para elaborar el currículo de una didáctica humanista. En Didáctica y Formación transidsciplinar. Pujol, Rajadell y Suanno (Coord.) Ed. Círculo Rojo, 2013.

MONTANÉ, A. Sobre justicia social y educación. En Montané, A. Sánchez Valverde, C. Derechos humanos y educación social. Alzira: Germania, 2013.

MORAES, M.C. Complejidad, Transdisciplinariedad y Educación: Algunas Reflexiones. Encuentros Multidisciplinares 9 (25), 2007. 4-13.

MORAES, M.C. Transdisciplinaridade, creatividades e educaçao. Fundamentos ontológicos e epistemológicos. Colaboraçao de Juan Miguel Batalloso Campinas, S.P. Papirus (Coleçao Práxis), 2015.

MORAES, M.C. Pensamento Ecossistêmico Educação, Aprendizagem e

Cidadania. En Educação Transdisciplinar: Escolas Criativas e Transformadoras. eduft.

Universidad Federal do Tocantins. Pinho, Maria José de (Org). Berenice Feitosa. 
Marilza, Vanessa Rosa Suanno, João Henrique Suanno, 2020.

MORALES, R., \& CABRERA, J. Competencias docentes transversales, el método de selección MiZona-CDT. REDU: Revista de Docencia Universitaria, 10(2), 2012. 75.

MOTTA, R. Educación, transdisciplinariedad y Filosofía. En Torre \& Pujol, (coord..) Educar con otra conciencia: una mirada ecoformadora y creativa de la enseñanza (No. 370 E24e). Edit. Davinci, 2009.

MORIN, E. Introducción al pensamiento complejo. Barcelona: Gedisa. 2004.

MURILlO, F. J. y Hernández, R. Hacia un concepto de Justicia Social. REICE. Revista Iberoamericana sobre Calidad, Eficacia y Cambio en Educación, 9(4), 7-23. 2011

MURILLO, F. J. y HERNÁNDEZ, R. Liderando Escuelas Justas para la Justicia Social. Revista Internacional de Educación para la Justicia Social, 3(2), 13-32. 2014.

LASZLO, E. El cosmos creativo. Hacia una ciencia unificada de la materia, la vida y la mente. Barcelona: Kairós. 1997.

LANDAU, E. El vivir creativo. Teoría y práctica de la creatividad. Barcelona: Herder (e.o.: 1987). 2002.

LÓPEZ, R. Desarrollos conceptuales y operacionales acerca de la creatividad. Chile. Cuadernos de Trabajo $n^{o} 1$. Universidad Central. Escuela de Ciencias de la Educación. 1995.

RAWLS, J. A theory of justice. Cambridge, MA: Harvard University Press [traducción al español (1978). Teoría de la Justicia. México: FCE]. 1971.

RAWLS, J. Justice as Fairness: A Restatement. Cambridge, MA: Belknap Press [traducción al español (2001). La justicia como equidad. Una reformulación. Madrid: Paidos Ibérica]. 2001.

SANTOS, B. Una epistemología del Sur: La reinvención del conocimiento y la emancipación social. México: Siglo XXI. 2009.

SEN, A. Desarrollo y libertad. Barcelona: Planeta. 2000.

SEN, A. (2010). La idea de la justicia. Madrid: Taurus. 2010. 
STERNBERG, R. Y LUBART, T. El concepto de creatividad. Perspectiva y paradigma”. En Handbook oh Creativity. Cambridge: Cambridge University Press. 1999.

SUANNO, M. V. \& CARDOSO, C. Resiliencia, adversidade criadora e educaçao. En Resiliencia, criatividades e innovaçao. Potencialidades transdisciplinares na educaçao. (Suanno, Ditrich \& Pujol (Org.)Goiana. UEG/Ed. América, 2013.

TORRE, S. de la. "Evaluar la creatividad docente. El profesorado creativo". En S. Torre y V. Violant (Coords.), Comprender y Evaluar la Creatividad, Vol. I. Málaga: Aljibe. 683-692. 2006.

TORRE, S. de la. Adversidad Creadora. Teoría y Práctica del Rescate de Potencialidades Latentes. Encuentros Multidisciplinares, 9 (31), 6-20. 2009a.

TORRE, S. de la. Escuelas creativas. Escuelas que aprenden crean e innovan. En Torre \& Pujol, (coord.) Educar con otra conciencia: una mirada ecoformadora y creativa de la enseñanza (No. 370 E24e). Edit. Davinci. 2009b.

TORRE, S. de la. Adversidad Creadora. Desarrollando otra Conciencia. En S. Torre y M Pujol (Coords.), Creatividad e innovación. Enseñar e investigar con otra conciencia. Madrid: Universitas. 19-34. 2010.

TORRE, S. de la. La Adversidad esconde un tesoro. Otra manera de ver la adversidad y la vida. Almería: Círculo Rojo. 2011.

TORRE, S. de la. Instituciones educativas creativas. Instrumento para valorar el desarrollo creativo de las instituciones creativas (VADECRIE). Almería: Círculo Rojo. 2012.

TORRE, S. de la. Movimiento de escuelas creativas. Haciendo parte de la historia y la transformación. En: Marlene Zwierewiez (Org.) Criatividad e inovaçao no ensino Superior: Experiencias latino-amiericanas e europeias em foco. (pp. 139-162). Blumenau: Nova Letra. 2013.

TORRE, S. de la \& SWIEREWICZ, M. Proyectos criativos ecoformadores. In: Marlene Zwierewiez \& Saturnino Torre (Org.) Uma escola para o século XXI:escolas creativas e resiliencia na educaçao. (pp. 153-176). Florianopolis: Insular. 2009.

TORRE, S. de la, PUJOL, M. A., \& SANZ, G. Transdisciplinariedad y ecoformación: una nueva mirada sobre la educación. Madrid: Universitas. Díaz-Barriga, F., Lule, M. d. L., Pacheco Pinzón, D. 2007. 
138 Polyphonía, v. 32/1, jan.-jun. 2021

Recebido em: 30 ago. 2020

Aceito em: 11 dez. 2020 